\title{
Evoked potentials and other guiding factors of conversion from radiologically isolated syndrome to definite multiple sclerosis

\author{
Serkan Demir ${ }^{1 *}$, Eyup Duzgun ${ }^{2}$, Cahit $\operatorname{Kafadar}^{1}$, Rifat Erdem Togrol ${ }^{1}$, Mehmet Guney Senol ${ }^{1}$, \\ Akin Cakir ${ }^{3}$
}

\begin{abstract}
Objective: Radiologically Isolated Syndrome (RIS) has become a popular subject recently with quite a number of follow-up and other clinical studies being done. A consensus on the approach to the patient with RIS is being tried to be established. The aim of our study was to assess the role of visual evoked potential (VEP) and somatosensory evoked potential (SEP) as a guiding factor for the conversion from Radiologically Isolated Syndrome (RIS) to definite Multiple Sclerosis (MS).

Material and methods: 49 RIS patients who were referred to GATA Haydarpasa Training Hospital Neurology Department between 2011-2015. All of the patients fullfillied the 2009 Okuda criteria for RIS and other differential diagnosis were excluded accordingly. VEP and SEP examinations made during the pre-MS RIS period were scanned retrospectively. For the VEP examination, the P100 latency and amplitudes, for the SEP examination, the P40 latency and amplitude was analysed.
\end{abstract}

Results: 49 patients were included in this study, the mean time of follow-up was 21,8 months. $63 \%$ of patients were female, while $37 \%$ were male. The mean age was 31,2 years. Among the four patients with abnormal SEPs, MS developed in three of them $(75 \%)$ over time. This is statistically significant $(\mathrm{p}=0.011)$. VEP and/or SEP was abnormal in 8 patients and MS developed in $4(50 \%)$ of those $(\mathrm{p}=0.017)$. The following factors have a positive statistically significant correlation with conversion to MS: Presence of active plaques ( $r=0.461$, $\mathrm{p}<0.001)$, presence of more than 9 plaques $(\mathrm{r}=0.287, \mathrm{p}=0.046)$, VEP and/or SEP pathologies $(\mathrm{r}=0.402$, $\mathrm{p}=0.004)$ and number of plaques $(\mathrm{r}=0.309, \mathrm{p}=0.031)$. The most important factor for the transformation is the presence of active plaque which increases the risk 8.1 -fold. The second important factor seems to be the presence of VEP and/or SEP abnormality, but this factor does not reach statistical significance.

Conclusion: In the conversion to MS risk from RIS, VEP- SEP examinations are important and should take its place in the follow-up of these patients.

Key words: Multiple syclorosis, Evoked potential, Radiological isolated syndrome

\section{Introduction}

As the imaging methods have become prevalent, more and more cases are being encountered with no specific complaints, symptoms or neurological examination findings but with lesions of their white matter in their magnetic resonance imagings (MRIs) that have been performed for various neurological complaints not implying multiple sclerosis (MS). This is usually called "asymptomatic MS" or nowadays more commonly, "Radiologically Isolated Syndrome (RIS)" $(1,2)$. It is known that some of these patients are known to turn into definite MS over the years. While RIS may lead to definite MS, the observed asymptomatic MRI lesions are lack of the pathological confirmation.
Evoked potential investigations (EPs) are used to evaluate a physiological system in real time. Physiological system here refers to sensorial afferent pathway functions (i.e visual, touching and hearing). Usually both latency and amplitude of the potential are evaluated and compared to normal values in these. The study to evaluate the visual pathways with the help of giving stimulations to one eye in the form of flash or changing checkerboard pattern is called "Visual Evoked Potential (VEP)". Sensory information is evaluated on the physiological level is evaluated by the "Somatosensory Evoked Potentials (SEP)". VEP and SEP show the real-time status of the visual and somatosensory afferent pathways function.

Received 28-05-2017 Accepted 10-06-2017 Available Online 30-06-2017

1 Sultan Abdulhamid Khan Training Hospital, Neurology, Istanbul, TR

2 Sisli Hamidiye Etfal Training Hospital, Ophtalmology, Istanbul, TR

3 Okmeydanı Training Hospital, Ophtalmology, Istanbul, TR

* Corresponding Author: Serkan Demir E-mail: drsrkndemir@gmail.com Phone: + 902165422020 
Although it has been removed from the revised McDonald criteria in 2010, VEP and SEP are still known to occupy a very important place in the diagnosis and follow-up of patients.

In this study, VEP and SEP examinations of patients were scanned before the "pre-MS" RIS period retrospectively, reviewed for any abnormality at this stage, and tried to determine whether there is a guiding factor for the transformation or not.

\section{Material and Methods}

The 49 patients who were hospitalized to GATA Haydarpasa Training Hospital Neurology Department between 2011-2015 whom, neurological symptoms not typical for MS, and with no neurological examination findings for MS, while having demyelinating lesions in their cranial MRI were included in the study. All of the patients fullfillied the 2009 Okuda criteria for RIS and differential diagnosis were excluded accordingly. None of the evaluated patients had any other medical condition that could create an abnormality in the electrophysiological studies.

\section{VEP test protocol}

The VEP were performed by using a MEDELEC TM Multimedia EMG/EP Synergy Monitoring System (London, United Kingdom) in abidance of the current guideline for pattern VEP of the International Societyfor Clinical Electrophysiology of Vision (3). Complete ocular examinations of anterior and posterior segments were done by the two ophthalmologists, and then patients went to VEP test.

VEP measurements were obtained with the patient wearing a visual aid, if needed and each eye tested separately. A steel needle recording electrode was placed at $\mathrm{Oz}$ ' ( $2 \mathrm{~cm}$ above the inion) and the reference electrode at Fz. Stimulation was performed according to standard protocols by checkerboard pattern reversal by using a computer screen at a distance of $1 \mathrm{~m}$ with a check size of 60 minutes of arc.

VEP test results assessed by two ophthalmologists and one neurologist. The mean P100 VEP latencies of the right and the left eyes of each patient were measured. According to laboratory normal values, the upper latency limit for the P100 latency was $120 \mathrm{~ms}$. P100 latency was considered abnormal if there is not any wave, latency longer than $120 \mathrm{~ms}$ in each of eyes or a difference equal or more than $6 \mathrm{~ms}$ between two eyes.

\section{SEP test protocol}

For SEP recordings, subjects lay on a couch in a warm and semidarkened room. All SEP examinations were made using MEDELEC TM Multimedia EMG/EP Synergy Monitoring System (London, United Kingdom). SEPs were elicited by electrically stimulating the posterior tibial nerves by superficial electrodes at the ankle on medial malleolus. The ground electrode was placed $10 \mathrm{~cm}$ distal to the active electode. Square wave stimuli of $0.2 \mathrm{~ms}$ duration were delivered via a bipolar surface stimulator with a frequency of $3 / \mathrm{sec}$. The stimulus intensity was adjusted to be slightly above the motor threshold, which in most cases was also twice the threshold for radiating paresthesia towards the toes.

The cortical potentials were recorded with electrodes placed on the head of the subject. One recording electrode (impedance below $5 \mathrm{kQ}$ ) was placed at the Cz' while another was placed at the Fpz (according the 10-20 system) as the reference electrode. The analysis time was $120 \mathrm{~ms}$, including also $10 \mathrm{~ms}$ of preanalysis. The amplifier bandpass was 100-2000 $\mathrm{Hz}$. Two averages of 500 trials each were obtained for each condition and printed out by the computer on a printer. It is well known that movement attenuates the early cortical SEPs, thus patients were asked to stay still during the procedure. For tibial SEP, latency and amplitude of the positive peak around $38-40 \mathrm{~ms}$ after the stimulating pulse (p40) was evaluated.

Tibial nerve SEPs were evaluated using established standards of our laboratory by two neurologist. (4). The criterion of a pathologic event in the SEP was defined as an increase of latency or a reduction of amplitude. Changes in SEP were considered pathological only if they were repeatable and sustained across at least two consecutive testings.

\section{Statistical analyses}

Statistical analyses were performed using SPSS software version 15 . The univariate analyses to identify variables associated with MS occurence during follow up, was investigated using Fisher's exact test, and chi square tests, where appropriate. Since the data were non-normally distributed and ordinals, the correlation coefficients and their significance were calculated using the Spearman test. For the multivariate analysis, the possible factors identified with univariate analyses were further entered into the logistic regression analysis to determine the independent predictors of MS occurance. Hosmer-Lemeshow goodness of fit statistics were used to assess model fit. A 5\% type-I error level was used to infer statistical significance.

\section{Results}

The 49 patients were included in this study which was designed as a retrospective cohort study. The mean time of follow-up was 21.8 months. $63 \%$ of patients were female, while $37 \%$ were male. The mean age was 31.2 years. Most frequent age range was 26-35 which is $39 \%$ of all the patients. The following factors have a positive statistically significant correlation with conversion to MS: Presence of active plaques ( $\mathrm{r}=$ $0.461, \mathrm{p}<0.001)$, presence of more than 9 plaques $(\mathrm{r}=$ $0.287, \mathrm{p}=0.046)$, VEP and/or SEP pathologies $(\mathrm{r}=$ $0.402, \mathrm{p}=0.004)$ 
The most common complaints at the time of referral was headache $(55 \%) .77 \%$ of the patients had more than two plaques. More than 9 plaques were detected in 21 cases $(\% 42.8) .7$ cases $(14.2 \%)$ had abnormal
VEP results while 4 cases $(8,1 \%)$ had abnormal SEP results. Among all the patients, 21 of the cases (42.8\%) developed MS over time. Demographic and clinical data of the patients are summarized in Table 1.

Table 1:Patient charecteristics

\begin{tabular}{|c|c|c|c|c|c|c|c|c|c|}
\hline Patient & Gender & Age & Symptoms & $\begin{array}{c}\text { Number } \\
\text { of } \\
\text { Plaques }\end{array}$ & $\begin{array}{l}\text { Gd (+) } \\
\text { plaque }\end{array}$ & $\begin{array}{l}\text { Spinal } \\
\text { plaque }\end{array}$ & $\begin{array}{c}\text { VEP } \\
\text { P100 } \\
\text { latan } \\
\text { ce }\end{array}$ & $\begin{array}{c}\text { SEP } \\
\text { P40 } \\
\text { latance }\end{array}$ & OKB \\
\hline 1 & $\mathrm{~F}$ & 18 & Syncope & 2 & - & - & $\mathrm{N}$ & $\mathrm{N}$ & $X$ \\
\hline $2 *$ & $\mathrm{~F}$ & 28 & Epilepsy & 5 & - & - & $\mathrm{N}$ & $\mathrm{N}$ & $\mathrm{Neg}$ \\
\hline 3 & $\mathrm{~F}$ & 38 & Backache & $>9$ & 1 & 1 & $\mathrm{~N}$ & $\mathrm{~N}$ & Pos \\
\hline 4 & $\mathrm{~F}$ & 29 & $\begin{array}{l}\text { Peripheral } \\
\text { Facial Palsy }\end{array}$ & 2 & 1 & - & $\mathrm{N}$ & $\mathrm{N}$ & $\mathrm{X}$ \\
\hline 5 & $\mathrm{M}$ & 22 & Headache & $>9$ & - & - & $\mathrm{N}$ & $\mathrm{N}$ & Neg \\
\hline 6 & $\mathrm{~F}$ & 42 & Headache & 3 & - & - & $\mathrm{N}$ & $\mathrm{N}$ & $\mathrm{X}$ \\
\hline 7 & $\mathrm{~F}$ & 34 & Headache & 3 & - & - & $\mathrm{N}$ & $\mathrm{N}$ & $\mathrm{X}$ \\
\hline 8 & $\mathrm{M}$ & 24 & Headache & 2 & - & - & $\mathrm{N}$ & $\mathrm{N}$ & $\mathrm{Neg}$ \\
\hline 9 & $\mathrm{~F}$ & 36 & Vertigo & $>9$ & 1 & 1 & $\mathrm{~N}$ & $\mathrm{~N}$ & Pos \\
\hline 10 & $\mathrm{M}$ & 30 & Headache & $>9$ & - & - & $\mathrm{N}$ & $\mathrm{N}$ & $\mathrm{X}$ \\
\hline 11 & $\mathrm{M}$ & 36 & Headache & $>9$ & & & $\mathrm{~N}$ & $\mathrm{~N}$ & $\mathrm{X}$ \\
\hline 12 & $\mathrm{~F}$ & 35 & Vertigo & $>9$ & - & - & $\mathrm{N}$ & $\mathrm{N}$ & $\mathrm{X}$ \\
\hline 13 & $\mathrm{~F}$ & 28 & Headache & $>9$ & - & - & $\mathrm{R}>$ & $\mathrm{N}$ & Neg \\
\hline $14^{*}$ & $\mathrm{~F}$ & 48 & Vertigo & $>9$ & 2 & 1 & $\mathrm{~N}$ & $\mathrm{R}>\mathrm{L}$ & Pos \\
\hline 15 & $\mathrm{~F}$ & 45 & Headache & $>9$ & - & - & $\mathrm{N}$ & $\mathrm{N}$ & $\mathrm{X}$ \\
\hline 16 & $\mathrm{~F}$ & 38 & Vertigo & 4 & - & - & $\mathrm{N}$ & $\mathrm{N}$ & $\mathrm{X}$ \\
\hline 17 & $\mathrm{~F}$ & 25 & Headache & 6 & - & - & $\mathrm{N}$ & $\mathrm{N}$ & Pos \\
\hline 18 & $\mathrm{~F}$ & 30 & Headache & $>9$ & - & - & $\mathrm{N}$ & $\mathrm{N}$ & $\mathrm{X}$ \\
\hline 19 & $\mathrm{~F}$ & 27 & Headache & 2 & - & - & $\mathrm{N}$ & $\mathrm{N}$ & $\mathrm{X}$ \\
\hline $20^{*}$ & $\mathrm{~F}$ & 35 & Vertigo & 4 & 1 & - & $\mathrm{L}>$ & $\mathrm{N}$ & Pos \\
\hline 21 & $\mathrm{~F}$ & 27 & Epilepsy & 6 & - & - & $\mathrm{N}$ & $\mathrm{N}$ & $\mathrm{X}$ \\
\hline $22 *$ & $\mathrm{M}$ & 27 & Headache & $>9$ & 2 & - & $\mathrm{N}$ & $\mathrm{N}$ & Pos \\
\hline $23 *$ & $\mathrm{~F}$ & 32 & Head Travma & $>9$ & 5 & - & $\mathrm{R}>$ & $\mathrm{L}>$ & $\mathrm{X}$ \\
\hline 24 & $\mathrm{~F}$ & 37 & Headache & $>9$ & - & - & $\mathrm{N}$ & $\mathrm{N}$ & Neg \\
\hline 25 & $\mathrm{~F}$ & 18 & Headache & 7 & - & - & $\mathrm{N}$ & $\mathrm{N}$ & $\mathrm{X}$ \\
\hline 26 & $\mathrm{M}$ & 21 & Tremor & 2 & - & - & $\mathrm{N}$ & $\mathrm{N}$ & $\mathrm{X}$ \\
\hline 27 & $\mathrm{~F}$ & 33 & Headache & 2 & - & - & $\mathrm{N}$ & $\mathrm{N}$ & $\mathrm{X}$ \\
\hline 28 & $\mathrm{M}$ & 41 & Headache & 2 & - & - & $\mathrm{N}$ & $\mathrm{N}$ & Neg \\
\hline $29 *$ & $\mathrm{~F}$ & 23 & Headache & $>9$ & 2 & 2 & $\mathrm{R} / \mathrm{L}>$ & $\mathrm{R} / \mathrm{L}>$ & Pos \\
\hline 30 & $\mathrm{M}$ & 21 & Headache & 5 & 1 & - & $\mathrm{N}$ & $\mathrm{N}$ & Neg \\
\hline $31 *$ & $\mathrm{~F}$ & 20 & Vertigo & 2 & - & - & $\mathrm{N}$ & $\mathrm{N}$ & $\mathrm{X}$ \\
\hline 32 & $\mathrm{M}$ & 21 & Head Trauma & 6 & - & - & $\mathrm{N}$ & $\mathrm{N}$ & $\mathrm{X}$ \\
\hline 33 & $\mathrm{~F}$ & 32 & Headache & $>9$ & - & - & $\mathrm{N}$ & $\mathrm{N}$ & $\mathrm{X}$ \\
\hline $34 *$ & $\mathrm{M}$ & 26 & Vertigo & $>9$ & - & 1 & $\mathrm{~N}$ & $\mathrm{~N}$ & Pos \\
\hline 35 & $\mathrm{~F}$ & 36 & Vertigo, Cramps & 2 & - & - & $\mathrm{N}$ & $\mathrm{N}$ & $\mathrm{X}$ \\
\hline 36 & $\mathrm{M}$ & 24 & Headache & 2 & - & & $\mathrm{N}$ & $\mathrm{N}$ & $\mathrm{X}$ \\
\hline 37 & $\mathrm{M}$ & 22 & Epilepsy & $>9$ & - & - & $\mathrm{R} / \mathrm{L}>$ & $\mathrm{R} / \mathrm{L}>$ & Pos \\
\hline 38 & $\mathrm{M}$ & 21 & Headache & 3 & - & - & $\mathrm{N}$ & $\mathrm{N}$ & $\mathrm{X}$ \\
\hline 39 & $\mathrm{~F}$ & 49 & Headache & 5 & - & - & $\mathrm{N}$ & $\mathrm{N}$ & $\mathrm{X}$ \\
\hline 40 & $\mathrm{~F}$ & 30 & Headache & 2 & - & - & $\mathrm{N}$ & $\mathrm{N}$ & $\mathrm{X}$ \\
\hline 41 & $\mathrm{M}$ & 22 & Headache & 5 & - & - & $\mathrm{N}$ & $\mathrm{N}$ & $\mathrm{X}$ \\
\hline 42 & $\mathrm{~F}$ & 42 & Headache & $>9$ & - & - & $\mathrm{N}$ & $\mathrm{N}$ & Neg \\
\hline 43 & $\mathrm{M}$ & 26 & Headache & $>9$ & - & - & $\mathrm{R} / \mathrm{L}>$ & $\mathrm{N}$ & $\mathrm{X}$ \\
\hline 44 & $\mathrm{~F}$ & 47 & Headache & 5 & - & - & $\mathrm{N}$ & $\mathrm{N}$ & $\mathrm{X}$ \\
\hline $45^{*}$ & $\mathrm{M}$ & 44 & Peripheral Vertigo & $>9$ & - & - & $\mathrm{N}$ & $\mathrm{N}$ & Pos \\
\hline 46 & $\mathrm{~F}$ & 55 & Peripheral Vertigo & $>9$ & - & - & $\mathrm{N}$ & $\mathrm{N}$ & $\mathrm{X}$ \\
\hline 47 & $\mathrm{M}$ & 21 & Peripheral Vertigo & 5 & - & - & $\mathrm{L}>$ & $\mathrm{N}$ & $\mathrm{X}$ \\
\hline 48 & $\mathrm{~F}$ & 35 & Peripheral Vertigo & 5 & 1 & - & $\mathrm{N}$ & $\mathrm{N}$ & $\mathrm{X}$ \\
\hline 49 & $\mathrm{M}$ & 31 & Uveitis & 4 & - & - & $\mathrm{N}$ & $\mathrm{N}$ & Pos \\
\hline
\end{tabular}

* the patients that converted to MS,

$\mathrm{N}$ : normal, R: right, L: left, $\mathrm{X}$ : patients with no CSF studies 
Table 2: Conversion rates of risc factors to MS

\begin{tabular}{lcccc} 
Risc factors & Did not develop MS & Developed MS & Total & p value* \\
\hline $\begin{array}{l}\text { Number of patients with VEP } \\
\text { abnormality, } \mathrm{n}(\%)\end{array}$ & $4(57 \%)$ & $3(42.8 \%)$ & $7(14.2 \%)$ & 0.075 \\
$\begin{array}{l}\text { Number of patients with SEP } \\
\text { abnormality, } \mathrm{n}(\%)\end{array}$ & $1(25 \%)$ & $3(75 \%)$ & $4(8.1)$ & 0.011 \\
$\begin{array}{l}\text { Number of patients with VEP and/or } \\
\text { SEP abnormality, n (\%) }\end{array}$ & $4(50 \%)$ & $4(50 \%)$ & $8(16.3 \%)$ & 0.017 \\
Active plaque & $5(50 \%)$ & $5(50 \%)$ & $10(20.4 \%)$ & 0.005 \\
Patients with $>9$ plaques & $15(71 \%)$ & $6(28.5 \%)$ & $21(42.8 \%)$ & 0.060 \\
\hline
\end{tabular}

*Fisher exact test.

Table 3: Spearman correlation analysis of the MS, active plaque, plaque number, $>9$ plaque number and age.

\begin{tabular}{ccccccc} 
& & $\begin{array}{c}\text { Presence of } \\
\text { Active plaque }\end{array}$ & $>9$ plaque & $\begin{array}{c}\text { VEP and/or SEP } \\
\text { negative }\end{array}$ & $\begin{array}{c}\text { Plaque } \\
\text { number }\end{array}$ & Age \\
\hline \multirow{2}{*}{ MS existence } & $\mathrm{r}$ & 0.461 & 0.287 & 0.402 & 0.309 & 0.084 \\
& $\mathrm{p}$ value & $<0.001$ & 0.046 & 0.004 & 0.031 & 0.566 \\
\hline
\end{tabular}

Table 4: Multiple logistic binary regression analysis.

\begin{tabular}{lccc} 
Risk factors & RR $^{*}$ & \%95 Confidence interval (CI) & p value \\
\hline Presence of active plaques & 8,103 & $(1.232-53.285)$ & 0.029 \\
Abnormal VEP or SEPs & 4,511 & $(0.562-36.244)$ & 0.156 \\
More than 9 plaques & 3,262 & $(0.428-24.839)$ & 0.254 \\
Age & 1,015 & $(0.904-1.141)$ & 0.797 \\
\hline
\end{tabular}

*RR: estimated relative risk shown by odds ratio and $95 \%$ confidence intervals.

Among the four patients with abnormal SEP, MS developed in three of them $(75 \%)$ over time. This is statistically significant $(\mathrm{p}=0.011)$. VEP and/or SEP was abnormal in 8 patients and MS developed in 4 $(50 \%)$ of those $(\mathrm{p}=0.017)$. Of the 10 patients with an active plaque, 5 of them $(50 \%)$ developed MS ( $p=$ 0.005).

Of the 7 patients with only an abnornal VEP response, conversion to MS was observed in only 3 (42.9\%) (p $=0.075)$. MS developed in 6 out of 21 patients $(28.6 \%)$ with more than 9 plaques $(p=0.060)$. Univariate analysis findings are summarized in the Table 2.

The following factors have a positive statistically significant correlation with conversion to MS: Presence of active plaques $(r=0.461, p<0.001)$, presence of more than 9 plaques $(\mathrm{r}=0.287, \mathrm{p}=$ $0.046)$, VEP and/or SEP pathologies $(\mathrm{r}=0.402, \mathrm{p}=$ $0.004)$ and number of plaques $(r=0.309, p=0.031)$. These all have a statistically significant positive lowto-moderate correlation. No significant statistical relationship between age and conversion to MS was found. $(r=0.084, p=0.566)$ (Table 3$)$.
When the relationship between the presence of abnormality in VEP and/or SEP and the number of plaques, and the presence of more than 9 plaques is evaluated, a positive low-to-moderate statistically significant correlation is calculated $(\mathrm{r}=0.309, \mathrm{p}=$ $0.031 ; \mathrm{r}=0.287, \mathrm{p}=0.046$; respectively). There is a mild positive statistically significant correlation between SEP deterioration and spinal plaques $(r=$ $0.392, \mathrm{p}=0.005)$.

In order to to calculate how effective these risk factors are in the transformation to MS, we have created a logistic regression model and we have seen that the most important factor for the transformation is the presence of active plaque. (Table 4) The presence of active plaque increases the risk 8.1 -fold. The second important factor seems to be the presence of VEP and/or SEP abnormality, but this factor does not reach statistical significance. Similarly, the presence of 9 or more plaques does not reach statistical significance.

In the power analysis using $\mathrm{G}$ power 3.1.9.2 belonging to this multiple binary logistic regression analysis, the power was found to be 0.934 . 


\section{Discussion}

Patients with MRI investigations made for any reason that is compatible with MS incidentally, while having no clinical data or medical clinical examination findings are termed or identified as RIS $(1,25,6)$. At this stage, there is no consensus on this clinical entity and no suggested treatment today $(5,7,8,9)$. None of the patients admitted in the RIS phase, which is included in our study were receiving a conventional MS treatment. Most common complaint among the RIS patients was identified to be headache in various studies as found in this study $(1,5,10)$.

RIS is a popular topic of recent times. 2009 Okuda criteria are used in the diagnosis of RIS. There have been a number of studies on this subject and some clinical follow-up work is being done. It is intended to achieve a consensus on the approach to these patients.

It is usually stated that while RIS may usually lead to definite MS, the observed asymptomatic MRI lesions most often than not lack pathological confirmation. Pathologically confirmed inflammatory demyelinating disease compatible with MS have been reported in a limited number of patients where the pathology of RIS has been described as "indistinguishable from classic MS pathology" (11). Of course, not all RIS patients develop clinically definite MS.

Findings consistent with RIS were observed in 23 out of 2783 psychiatric patients in a study made in 1996 (12). Studies have been published in which demyelinating lesions were reported in less than $0.5 \%$ of the radiological scans of asymptomatic patients without any complaints (13-15). RIS is seen more frequently in people with family members with MS than those without (16). Data of the twin studies also support this statement. It seems that genetic predisposition is a risk factor.

Neurological symptoms develop during follow-up in proportion of patients at different ratios in various studies. Approximately in two-thirds of the patients develop radiographic progression within the first 5 years. When the number of lesions in the MRI is high $(>9)$, gadolinium (Gd) enhancement of the asymptomatic lesions is present and in particular if cervical cord lesions are seen, clinical conversion rate is higher $(2,5,6,7,17,18,19)$. In our study, 8 of 49 patients had subsequent neurological episodes during the follow-up period, and thus began to be followed by a diagnosis of Clinically Isolated Syndrome (CIS) MS accordingly. 6 of these 8 patients had more than 9 plaques at the beginning and 5 had Gd-enhancing active plaques at the time of RIS diagnosis. All the patients are still being followed up and the subsequent revisions of this text in the following years, the diagnosis of the patients that are still being followed up with RIS diagnosis and Gd-enhancing lesions arouses interest. Thus whether or not a treatment is feasible for RIS patients that are highly probable to turn into definite MS over the years is still debatable $(7,9)$.

MS is perhaps the most common area of use in the practice of neurology the EPs are used today. They are especially utilized in patients when MS is considered, where the sign and symptoms are not adequate or definite, while the electrophysiological conduction defects due to the subclinical lesions in the background are shown (20-22). Although they are not required in the 2010 revised McDonald criteria, they are still frequently used in the follow-up of patients.

VEP is extremely sensitive in showing lesions in the anterior visual pathways. While it gives objective information on acute optic neuritis, it also gives an idea by providing data on the chronic period. Therefore, one can conclude that VEP examination is more sensitive and less expensive compared to MRI for showing optic nerve lesions, and a normal VEP examination can more or less virtually rule out the possibility of an optic nerve and/or chiasm lesion in a patient (22-24). The superiority of VEP examination to other methods for showing early demyelination of the optic nerve and follow-up, including OCT and retinal nerve fiber examinations have been are reported in some studies (24).

SEP are obtained from appropriate regions by stimulating a sensory nerve anywhere in the body, after giving mechanical, electrical or magnetic stimuli. Unlike EEG, they are not affected by general anesthesia or sedatives (25). Most frequently central responses obtained after mixed nerve stimulation (median and/or posterior tibial nerve) are evaluated. Pathological processes in the central nervous system that cause SEP abnormalities are most frequently in the spinal cord. The main purpose of this investigation in demyelinating diseases is to show "silent" lesions. Lesions that belong to a specific region of the nervous system with no evidence in clinical examination or history can be detected with SEP. In demonstrating a "silent" lesion, SEP is almost as sensitive as VEP $(20,26)$. The main pathology in MS is demyelination and axonal degeneration. Accordingly, slowing of the transmission in nerve fibers occurs, this transmission becomes dispersed or is completely blocked. This functional disorder is reflected in the EPs. The median and ulnar SEP studies are rarely abnormal in MS patients, while the studies made by the stimulation of lower extremity have pathological or abnormal results more often. This situation is explained by the longer way in the spinal cord the somatosensory pathways go from the lower extremities and are therefore more likely to come across an area of demyelination (27). Sometimes even in patients with the definite diagnosis of MS, EPs are known to be used to "confirm the diagnosis", in reality to show the lesions with no radiographic or clinical findings. On the other hand, EPs may be used serially to follow-up patients. This is applicable for both SEP and for VEP. Tsao et al. have used SEP in their study for monitoring neuromyelitis 
optica prognosis and have reported it to be beneficial (28).

There are very few studies that have examined the relationship of EPs with RIS. In a study that have compared CIS and RIS patients who have been examined with oligoclonal bands, antinuclear antibodies and VEP has shown that VEP pathology is still an important parameter to show demyelination episode. RIS patients have been determined to have a lower percentage of OCB positivity and VEP abnormality compared to CIS patients, which implies that every RIS patient will not eventually become a CIS, or eventually MS patient (24).

The small number of patients and the lack of the number of RIS patients who eventually turned to CIS or MS are the weak points of that study. In another study in which 70 patients were followed-up prospectively, abnormal VEP, younger age, and Gd enhancement on follow-up MRI were more frequent in clinically definite MS than in MS determined by MRI (29). In a review of American Academy of Neurology, it is reported, based on various publications, that those with a probable MS and abnormal VEP investigations have 2.5-9 times more probability to develop clinically deifinite MS compared to those with normal VEP studies. Likewise, in the same review it can be seen that some studies have reported 2.4-3.9 times more likelihood to develop clinically definite MS in those with SEP abnormalities; but several other studies have not replicated this finding (30).

Our opinion is that all patients with RIS should be evaluated with EPs. Because, RIS patients with an abnormal VEP or SEP abnormality have a higher risk of having an attack and developing CIS/MS according to the results of this study. The presence of active plaque has been found to be most important factor for convertion to MS. The second important parameter is abnormal VEP/SEP. This is even more valuable and significant than the number of plaques.

On the other hand, these patients should also be serially followed-up with EPs. This is true for both SEP and VEP. EPs can reveal lesions simulating that can not be detected with MRI (25). If an abnormal response from a patient that is not having an attack due to a silent lesion is obtained, this abnormality is expected to continue. Similarly, if an abnormal response due to a silent lesion from an asymptomatic patient with only radiological findings (RIS) is obtained, this abnormality is expected to continue. This rules out the technical problems and mistakes. Additionally, serial follow-up is helpful in monitoring the development of disease, determining prognosis and demonstrating new areas that are being affecting during the progression of the disease, i.e. new lesions.

There are some limitations of our work. Follow-up time is less than 2 years and our study is retrospective. We didn't use median SEP study on our patients.

\section{Conclusion}

Finally, we believe that all patients with RIS should be evaluated with EPs and followed-up serially with them. In order to understand the exact place of EPs in the follow-up of RIS, greater series with longer period of follow-up is needed.

Conflict of Interest: The authors declare no potential conflicts of interest with respect to the research, authorship, and/or publication of this article.

Author's Contributions: SD: Examination of patients, collecting of data, writing of manuscript, Revision of Article, ED, CK, RET, MGS, AC: Examination of Patients, Collecting of Data

Ethical issues: All Authors declare that Originality of research/article etc... and ethical approval of research, and responsibilities of research against local ethics commission are under the Authors responsibilities. The study was conducted due to defined rules by the Local Ethics Commission guidelines and audits.

\section{References}

1. Lebrun C, Cohen M, Chaussenot A, Mondot L, Chanalet S. A prospective study of patients with brain MRI showing incidental t2 hyperintensities addressed as multiple sclerosis: a lot of work to do before treating. Neurol Ther. $2014 ; 3(2): 123-32$

2. Etemadifar M, Janghorbani M, Koushki MM, Etemadifar F, Esfahani MF. Conversion from radiologically isolated syndrome to multiple sclerosis. Int J Prev Med. 2014 ;5(11):1379-86.

3. Odom JV, Bach M, Brigell M, Holder GE, McCulloch DL, Tormene AP, Vaegan. ISCEV standard for clinical visual evoked potentials (2009 update). Doc Ophthalmol. 2010;120:111-9.

4. Togrol E, Gurtekin Y, Saracoğlu M, Akyatan N Somatosensory Evoked Potentials \& Correlations With Gender, Age And Height. 9th European Congress of Clinical Neurophysiology, Ljubliana Electroencephalography and Clinical Neurophysiology, 1998; $106: 77$

5. Granberg T, Martola J, Kristoffersen-Wiberg M, Aspelin P, Fredrikson S. Radiologically isolated syndrome--incidental magnetic resonance imaging findings suggestive of multiple sclerosis, a systematic review. Mult Scler. 2013 ;19(3):271-80.

Okuda DT, Mowry EM, Beheshtian A, Waubant E, Baranzini SE, Goodin DS, Hauser SL, Pelletier D. Incidental MRI anomalies suggestive of multiple sclerosis: the radiologically isolated syndrome.Neurology. 2009 ;72(9):800-5.

7. De Stefano N, Siva A.The radiologically isolated syndrome dilemma: just an incidental radiological finding or presymptomatic multiple sclerosis? Mult Scler. 2013 ;19(3):257-8 
8. Yamout B, Alroughani R, Al-Jumah M, Goueider R, Dahdaleh M, Inshasi J, et al. Consensus recommendations for the diagnosis and treatment of multiple sclerosis: the Middle East North Africa Committee for Treatment and Research In Multiple Sclerosis (MENACTRIMS).Curr Med Res Opin. 2015 ;31(7):1349-61

9. Tornatore C, Phillips JT, Khan O, Miller AE, Barnes CJ. Practice patterns of US neurologists in patients with CIS, RRMS, or RIS. Neurol Clin Pract. 2012 ;2(1): 48-57.

10. Azevedo CJ, Overton E, Khadka S, Buckley J, Liu S, Sampat $M$, et al. Early CNS neurodegeneration in radiologically isolated syndrome. Neurol Neuroimmunol Neuroinflamm. $2015 ; 2(3): \mathrm{e} 102$

11. Keegan M., Guo Y., Okuda D., Siva A., Pelletier D., Kantarci O., Lucchinetti C., Lebrun Frenay C. Radiologically Isolated Syndrome: Pathologically Defined as Demyelinating Disease; Poster presented in the Annual meeting of AAN, P1.391, April 16, 2016

12. Lyoo IK, Seol HY, Byun HS, Renshaw PF. Unsuspected multiple sclerosis in patients with psychiatric disorders: magnetic resonance imaging study. J Neuropsychiatry Clin Neurosci 1996;8(1):54-9

13. Katzman GL, Dagher AP, Patronas NJ. Incidental findings on brain magnetic resonance imaging from 1000 asymptomatic volunteers. JAMA 1999;282(1):36-9

14. Weber $F$, Knopf $H$. Incidental findings in magnetic resonance imaging of the brains of healthy young men. $\mathrm{J}$ Neurol Sci 2006;240(1-2):81-4

15. Hartwigsen G, Siebner HR, Deuschl G, Jansen O, Ulmer S. Incidental findings are frequent in young healthy individuals undergoing magnetic resonance imaging in brain research imaging studies. J Comput Assist Tomo 2010;34(4):596-600

16. Gabelic T, Ramasamy DP, Weinstock-Guttman B, Hagemeier J, Kennedy C, Melia R, et al. Prevalence of Radiologically Isolated Syndrome and White Matter Signal Abnormalities in Healthy Relatives of Patients with Multiple Sclerosis. AJNR Am J Neuroradiol. 2014 Jan;35(1):106-12.

17. Brex PA, Ciccarelli O, O'Riordan JI, Sailer M, Thompson AJ, Miller DH. A longitudinal study of abnormalities on MRI and disability from multiple sclerosis. N Engl J Med. $2002 ; 346(3): 158-64$

18. Lana-Peixoto MA. How much radiologically isolated syndrome suggestive of multiple sclerosis is multiple sclerosis? Arq Neuropsiquiatr. $2012 ; 70(1): 2-4$
19. Okuda DT, Siva A, Kantarci O, Inglese M, Katz I, Tutuncu $\mathrm{M}$, et al. Radiologically isolated syndrome: 5-year risk for an initial clinical event. PLoS One. 2014;9(3):e90509.

20. Fischer C, André-Obadia N, Mauguière F. Diagnostic criteria of multiple sclerosis: electrophysiological criteria. Rev Neurol (Paris). 2001 ;157(8-9 Pt 2):974-80.

21. Kjaer M. Evoked potentials. With special reference to the diagnostic value in multiple sclerosis.Acta Neurol Scand. 1983;67(2):67-89.

22. Kjaer M. Brain stem auditory and visual evoked potentials in multiple sclerosis. Acta Neurol Scand. $1980 ; 62(1): 14-9$.

23. Leocani L, Comi G. Clinical neurophysiology of multiple sclerosis. Handb Clin Neurol. 2014;122:671-9.

24. Comi G, Leocani L, Medaglini S, Locatelli T, Martinelli V, Santuccio G, Rossi P. Measuring evoked responses in multiple sclerosis. Mult Scler. 1999;5(4):263-7.

25. Sand T, Kvalay MB, Wader T, Hovdal H. Evoked potential tests in clinical diagnosis. Tidsskr Nor Laegeforen nr. 9, 2013; 133(9): $960-5$

26. Loncarević N, Tirić-Campara M, Mulabegović N Somatosensory evoked cerebral potentials (SSEP) in multiple sclerosis. Med Arh. 2008 ;62(2):80-1.

27. Slimp JC, Janczakowski J, Seed LJ, Kraft GH. Comparison of median and posterior tibial nerve somatosensory evoked potentials in ambulatory patients with definite multiple sclerosis. Am J Phys Med Rehabil. 1990 ;69(6):293-6.

28. Tsao WC, Lyu RK, Ro LS, Lao MF, Chen CM, Wu YR, et al. Clinical Correlations of Motor and Somatosensory Evoked Potentials in Neuromyelitis Optica. PLoS One. 2014; 9(11): e113631.

29. Lebrun C, Bensa C, Debouverie M, Wiertlevski S, Brassat $\mathrm{D}$, de Seze J, et al. Association between clinical conversion to multiple sclerosis in radiologically isolated syndrome and magnetic resonance imaging, cerebrospinal fluid, and visual evoked potential: follow-up of 70 patients. Arch Neurol. 2009 ;66(7): 841-6

30. Gronseth GS., Ashman EJ. Practice parameter: The usefulness of evoked potentials in identifying clinically silent lesions in patients with suspected multiple sclerosis (an evidence-based review): Report of the Quality Standards Subcommittee of the American Academy of Neurology. Neurology. 2000; 54(9); 1720-5 